\title{
CryProcessor: a novel tool for mining Cry toxins in Bacillus thuringiensis sequencing data
}

\author{
Kirill Antonets \\ Laboratory for Proteomics of Supra- \\ Organismal Systems \\ All-Russia Research Institute for \\ Agricultural Microbiology \\ Saint Petersburg State University \\ St. Petersburg, Russia \\ k.antonets@arriam.ru \\ Anton Shikov \\ Laboratory for Proteomics of Supra- \\ Organismal Systems \\ All-Russia Research Institute for \\ Agricultural Microbiology \\ Saint Petersburg State University \\ St. Petersburg, Russia \\ a.shikov@arriam.ru
}

\author{
Anton Nizhnikov \\ Laboratory for Proteomics of Supra- \\ Organismal Systems \\ All-Russia Research Institute for \\ Agricultural Microbiology \\ Saint Petersburg State University \\ St. Petersburg, Russia \\ a.nizhnikov@arriam.ru
}

\begin{abstract}
Bacillus thuringiensis is a gram-positive bacterium, which produces very diverse class of protein toxins, Cry. Precise annotation of these toxins in the bacterial genomes is a very challenging problem due to the sequence similarity between different toxins can be lower than $40 \%$. Here we describe a novel tool, CryProcessor, for searching Cry toxins in protein sequences and in raw sequencing reads.
\end{abstract}

Keywords - Bacillus thuringiensis, Cry toxins, data mining

\section{Motivation and aim}

Bacillus thuringiensis is a gram-positive bacterium, widely used in agriculture, because it is a natural pathogen of insects. This species of bacteria produces very diverse class of protein Cry toxins, which are highly specific against particular species of insects. To date, more than 700 different Cry toxins are described, which can share less than $40 \%$ sequence similarity. This makes the search for Cry toxins, particularly the new ones, in bacterial genomes very difficult. Here we present a novel tool for searching Cry toxin sequences.

\section{Methods}

CryProcessor tool is based on several HMM for each domain of Cry proteins. The pipeline is written in Python3.7 and is able to find Cry toxin sequences in the sets of protein sequences or in the raw Illumina reads. It also provide domain coordinates for found toxin sequences. Detailed information is available at https://github.com/lab7arriam/cry_processor. Web-version of the tool is accessible at https://lab7.arriam.ru/tools/cry_processor.

\section{Results}

We have tested our tool, CryProcessor in all modes with protein sequences of all available $B$. thuringiensis genome assemblies, and also with several SRA sequencing data. It has been shown to be more sensitive and fast comaparing with the existing analogue, BtToxin_scanner [1]. It has been also able precisely to predict the domain coordinates of the found toxins, unlike BtToxin_scanner.

\section{Acknowledgment}

This work was supported by the Russian Science Foundation (grant No. 18-76-00028).

\section{References}

[1] Ye W., Zhu L., Liu Y., Crickmore N., Peng D., Ruan L., Sun M. “Mining new crystal protein genes from Bacillus thuringiensis on the basis of mixed plasmid -enriched genome sequencing and a computational pipeline". Appl. Environ. Microbiol., vol. 78, pp.4795-4801, 2012 